\title{
The role of olfactory cues in position learning in the gerbil
}

\author{
LEONARD BROSGOLE \\ St. John's University, Jamaica, New York 11439
}

Four groups of mature male gerbils were trained to position in a Y maze. Two groups (1 and 2) ran in a uniformly gray maze. Color was added to the start- and goalboxes for the other two groups ( 3 and 4$)$. The walls and doors of the startbox were washed prior to each trial for Groups 1 and 3 , so as to remove the possible deposit of chemical cues. The elimination of olfactory stimuli in the start had a devastating effect on the first group, which ran in the uniform maze. When color was added (Group 3), there was a marked increment in performance. These data indicated that stimuli in the start are used to cognitively structure a maze. When preferred olfactory cues are omitted, the gerbil utilizes visual stimuli.

Both the male and female gerbil deposit a pheromone in their surround by marking low-lying objects with a ventral, sebaceous scent marking gland. While scent marking has been associated with dominance and territoriality in the male (Thiessen, 1973; Thiessen, Lindzey, Blum, \& Wallace, 1970), it has been linked to maternal behavior in the female. It has been related to exploratory behavior in both sexes, however, and sebum has been found to be an attractant (Thiessen, Regnier, Rice, Goodwin, Isaacks, \& Lawson, 1974). Such findings have vast implications for performance in an instrumental task, for it is possible that sebum may be utilized in establishing olfactory trails, thereby promoting correct performance.

In a recent study, Brosgole, Neylon, and Ulatowski (1976) indicated that the exploratory behavior of sexually immature females was restricted almost exclusively to the startbox of a symmetrical Y maze. Such behavior included apparent scent marking with saliva and urine and the rubbing of the abdomen against the base of the walls and doors of the box. This would imply that scent marking may not be used as a means of getting to a goal-that the animal may not be pulled blindly to its reward by the delectably sweet smell of sebum or urine. As opposed to creating a chemical trail, it would appear that olfactory stimuli may serve to differentiate a maze, enabling the goal to be structured relative to a designated start position. In other words, it is entirely possible that olfaction may facilitate the cognitive structuring of a maze in the absence of any other available cues.

\section{METHOD}

\section{General}

In the following study, sexually mature males were trained to position in a symmetrical $Y$ maze, which was painted a uniform gray. In half the cases (Group 1), the walls and doors of the startbox were cleansed with a $5 \%$ solution of $\mathrm{NaHCO}_{3}$ prior to each run to eliminate any odor of sebum. The remaining half (Group 2) was run through the procedure with no such cleansing. Thus, we were able to assess the effect of destıoying olfactory stimuli in the start upon position learning in the gerbil. The entire design was repeated on two additional groups ( 3 and 4 ), with color used to differentiate the start and goals. Because the gerbil is a highly visual animal, we expected the introduction of color to negate any effect of cleansing.

\section{Subjects}

Thirty-two male Mongolian gerbils, strain Mon/tum, were used. They were at least 20 weeks of age at testing and were kept on an ad-lib diet of pellets and water.

\section{Apparatus}

A symmetrical $\mathrm{Y}$ maze was used. This device was described in detail by Brosgole and Lepak (1976) and Brosgole et al. (1976). In brief, it was constructed of $1 / 2$-in. plywood with the three arms intersecting at $120 \mathrm{deg}$ angles. Each arm was 3 in. wide $x 10$ in. high $\times 13$ in. long, with the last 4 in. of the arms accommodating removable start- and goalboxes. The start and goals were 4 in. long $x 3$ in. wide and were made of Masonite. The boxes contained flap-like leather doors that were hinged from above, so that their interiors could not be seen from the alleys of the maze.

The essential feature of the maze was that the animals performed to escape from heat that was being applied to the paws. The floors of the maze were constructed of $1 / 8$-in.-thick aluminum plate. The plates were heated from below with nichrome coils that were powered by a variable ac regulated supply.The floors of the start and alleys were adjusted to $45^{\circ} \mathrm{C}$ and the negative goal was $50^{\circ} \mathrm{C}$. The floor of the positive goal was at room temperature. The entire device sat upon a lazy susan, enabling the maze to be randomly rotated from trial to trial. This was done to prevent the animals from orienting to stimuli within the testing room.

The interior of the maze was painted a uniform flat gray of approximately $30 \%$ reflectance. The walls and doors of the start and goals were coated with the same gray paint for Groups 1 and 2. As mentioned earlier, colored boxes were used for Groups 3 and 4 . One box was black, the other white, and the third consisted of $2 \frac{1}{2}$-in.-wide black and white vertical stripes. While the inside of the door coincided with the color of the box, the outside was flat gray. Thus, the color of the box could not be detected from the alley. One of the boxes served as the start and the other as the goals (one positive and the other negative). The color of the start and goals was counterbalanced over animals in each group.

\section{Procedure}

The animals ran 30 trials a day. After being placed in the 
startbo\%, the animals were required to make a position response (a right or left turn) to escape from heat. The positive goal was to the left for half the animals in each group and to the right for the remaining half. The animals were detained in the goalbox of their choice for $10 \mathrm{sec}$. Upon being removed from the goalbox, the maze was rotated for the start of the next trial. Training continued until the animal reached a $90 \%$ criterion, i.e., at least 27 correct responses on a given day.

\section{Groups}

Each group consisted of eight animals. Groups 1 and 2 ran in a uniformly gray maze, while colored boxes were used for 3 and 4. For Groups 1 and 3, the walls and doors of the start were washed prior to each trial with a $5 \%$ solution of $\mathrm{NaHCO}_{3}$ and distilled water. This was not the case for Groups 2 and 4.

\section{RESULTS AND DISCUSSION}

The mean number of errors to criterion was $13.1,4.9$, 5.6, and 4.6 for Groups $1-4$, respectively. The data were subjected to a completely randomized block factorial design. There was a significant two-way interaction $(F=4.22, \mathrm{df}=1 / 28, \mathrm{p}<.05)$. Subsequent simple effects indicated that Group 1 committed significantly more errors to criterion than Group $2(\mathrm{~F}=357.04, \mathrm{df}=1 / 28$, $\mathrm{p}<.01$ ). In the same fashion, Group 3 made significantly more errors than Group $4(\mathrm{~F}=6.64, \mathrm{df}=1 / 28, \mathrm{p}<.05)$. Thus, the cleansing procedure seemed to have generated more errors regardless of the presence or absence of color. The question may be raised as to whether the animals' performance was negatively affected simply by the wetting of the walls of the startbox, as opposed to the actual removal of chemical cues. It was reasoned that, if the wetting alone was interfering with performance, then Groups 1 and 3 should have been more or less equivalent. However, Group 1 committed reliably more errors than Group $3(\mathrm{~F}=285.34, \mathrm{df}=1 / 28, \mathrm{p}<.01)$. It would seem, then, that the cleansing procedure was effective in removing odors from the start. This drastically impaired the animals' ability to structure and, hence, master the maze. It is as if they had to rely almost completely on response-produced stimuli. The animals were able to shift cues, however, with the addition of color, so that there was a substantial increment in performance.
It may be contended that color was added to the goals as well as the start, thereby making the maze substantially easier to learn. Such an argument would explain the difference between Groups 1 and 3. It would logically follow, then, that Group 4 should have committed fewer errors than Group 2. However, this was not the case $(\mathrm{F}=.34, \mathrm{df}=1 / 28)$, indicating that performance was not at all positively affected by the presence of visual stimuli in the goals.

It must be remembered that the washing was restricted to the walls and doors of the start. The floors, the alleys, and the goals were untouched. All groups had an equal opportunity to deposit and utilize trails in the maze, which would have equalized their performance. Our data indicated this was not done.

In conclusion, it would appear that chemicals are deposited on the walls and doors of the startbox of a maze. They serve to differentiate the start from the balance of the maze, enabling the maze to be structured cognitively and learned. The removal of these chemicals seems to disrupt the formation of such a map and negatively affects performance. Once removed, however, the chemicals can be replaced for the most part by visual cues.

\section{REFERENCES}

Brosgole, L., \& Lepak, C. Associative symmetry: I. Position learning in the gerbil. Bulletin of the Psychonomic Society, 1976, 7, 99-102.

Brosgole, L., Neylon, A., \& Ulatowski, P. Associative symmetry: Il. Further studies on position learning in the gerbil. Bulletin of the Psychonomic Society, 1976, 7, 117-120.

Thiessen, D. D. Footholds for survival. American Scientist, 1973, 61, 346-351.

Thiessen, D. D., Lindzey, G., Blum, S. L., \& Wallace, P. Social interactions and scent marking in the Mongolian gerbil (Meriones unguiculatus). Animal Behaviour, 1970, 19. $505-513$.

Thiessen, D. D., Regnier, F. E., Rice, M., Goodwin, M., IsAACKS, N., \& LAWSON, N. Identification of a ventral scent marking pheromone in the male Mongolian gerbil (Meriones unguiculatus). Science, 1974, 184, 83-85.

(Received for publication June 14, 1976.) 